\title{
Optimum Performance Bounds of Routing Protocols for VANET through Realistic Fading Channel
}

\author{
Aarja Kaur \\ Student, CSE Department \\ GNDU, Regional Campus \\ Jalandhar, India
}

\author{
Jyoteesh Malhotra \\ Head, CSE and ECE Department \\ GNDU, Regional Campus \\ Jalandhar, India
}

\begin{abstract}
Vehicular-Ad hoc Networks have the potential to achieve high levels of safety, efficiency and comfort on road. VANET is a campaign towards Intelligent Transport System (ITS). VANET applications are time critical and the QoS constraints require the designing and evaluation of routing protocols to be done in realistic environment. The main purpose of this paper is to simulate the routing protocols, AODV, OLSR and ZRP and to explore the impact of radio propagation models, Two Ray Ground and Nakagami in terms of QoS parameters. Extensive simulations are carried out using NS-2 and the behaviour of protocols is studied at different speeds under the influence of propagation models.
\end{abstract}

\section{General Terms}

Vehicular Ad-hoc Network, Radio Propagation Model, Protocol

\section{Keywords}

Two Ray Ground, Nakagami

\section{INTRODUCTION}

The concerns for road safety and traffic efficiency resulted in the emergence of Vehicular Ad-hoc Networks [4]. VANET is a communication paradigm that allows vehicles to exchange real time information that can assist drivers to avoid situations like traffic congestion, accidents etc. VANET supports short range wireless links without using centralized scheduling and can be categorized into V2V and V2I communication [10]. IEEE $802.11 \mathrm{p}$ standard was developed by enhancing the existing IEEE 802.11 to provide the QoS support for VANET based applications that aim to provide Intelligent Transport Systems. VANET has enough resources i.e. battery backup, high end on-board processing units, location monitoring system. But still VANET routing is a critical task because they have to face different node movements and densities, disconnections in different scenarios like city, urban, highway, rural etc [2]. VANET applications are mostly timecritical and reliable communication is necessary. The routing protocols in order to be applicable in VANET, should be able to adapt to the variations in the network and still show a desirable performance. For facilitating reliable communication among vehicles, it is necessary to evaluate the performance of the routing protocols in conditions that will actually prevail in reality. Mobility pattern, number of vehicles, their speed, and radio channel are some of the factors that must be considered when studying VANET as they significantly effect the operation of protocols [3]. MAC is also a key issue in the design of VANETs.

In our previous work [9], we had programmed two back-off methods in the MAC $802.11 \mathrm{p}$ to provide a solution for contention adaptability. Comparisons were done among the default back-off algorithm and the introduced algorithms. From the results, it was clear that the modification of the contention window size by Modified Back-off Algorithm (MBA) turned out to be better than the default back-off method in terms of throughput, packet delivery ratio, routing load and end to end delay. This work is an extension of the previous work. In this paper, we try to analyze the effects of radio channel on the performance of well known protocols

The main contribution of this paper is to study the behaviour of topology based routing protocols, one from each category of reactive, proactive and hybrid. The performance is evaluated considering two scenarios with vehicle speed of 10 $\mathrm{m} / \mathrm{s}$ and $20 \mathrm{~m} / \mathrm{s}$. Results are calculated for throughput, packet delivery ratio, routing load and end to end delay for each protocol with Two - Ray Ground and Nakagami propagation models.

The remainder of the paper is organized as follows. Section 2 discusses briefly the propagation models that are used in this paper. Section 3 describes the related researches done in evaluating the performance using propagation models. Section 4 presents the simulation environment. Section 5 analyzes and discusses the results obtained. Section 6 finally concludes the paper.

\section{PROPAGATION MODEL}

Radio propagation models are used to estimate the strength of the wireless signal received. They deal with the cross layer operations. At the physical layer, each packet is marked using a receiving signal threshold value which is further verified at MAC layer and packet is dropped if its receiving signal power is less than the threshold value. A number of radio propagation models have evolved for VANET [1].In this paper, we have considered two propagation models ,Two Ray Ground and Nakagami.

\subsection{Two Ray Ground}

Most research studies perform simulations using Two Ray Ground propagation model [2].It assumes multipath ground reflection [6].It takes into account the superposition of line of sight signal and the signal reflected from the ground. Unobstructed environment is assumed in two ray ground model [5].This model is not perfect for VANET scenarios [1] [3].

\subsection{Nakagami}

It is a probabilistic radio propagation model [4] and is well suited for VANET [1] because of its ability to reflect the realistic conditions of fading. Wireless communication channel can be configured from low to moderate to highly intense fading channel to represent reality [2]. 


\section{RELATED WORK}

Various research studies have analysed the performance of routing protocols in the presence of mobility models and propagation models. Some of the previous research done in comparing the radio propagation models are given in brief.

The authors in [1] have simulated AODV protocol using NS-2 in order to investigate the performance of four propagation models, Two Ray Ground, Free Space Model, Shadowing model and Nakagami. Mobility traces are generated using VANET MOBISIM. The results show that among the four models, Nakagami appears to be the most preferable model for VANET. It proves to be suitable according to number of nodes and has minimum packet loss.

[2] is another effort in assessing the impact of Two Ray Ground and Nakagami Model on the operation of protocols in VANET. AODV and OLSR protocols are evaluated in urban scenario. Packet Delivery ratio and end to end delay are calculated for Two Ray Ground and Nakagami by varying the number of nodes and connections. The authors conclude that Nakagami is more realistic and better suited in dynamic VANET scenarios.

The authors in [3] highlighted the importance of using adequate radio propagation and mobility models are explained in detail, features of each are presented.

[4] Focuses on the significance and the impact of radio channel modelling on the performance of wireless communication systems.

The deviations in the results obtained by various propagation models, motivated the authors in [5] to validate the models under variable network load. The authors have proposed a propagation model using dual slope path loss model and Nakagami Fading which is suitable for V2V communication on highways. The proposed model proves to be more realistic than the counterparts.

[6] Presents the impact of radio propagation models on the number of warning messages disseminated and the presence of blind vehicles. Two Ray Ground propagation model is compared with Distance Attenuation Model and Building model. Authors proposed a new model, Building and Distance Attenuation Model. Evaluations of the models are done by varying vehicle density and building size. The proposed model reflects reality in an accurate way.

Authors in [7] carried out intensive simulations to determine the safety beacon loss rate in VANETs Beacon loss rate was analysed with Two Ray Ground and Nakagami propagation models as a function of communication range and beacon interval.

Realizing the remarkable impact that the propagation models can exhibit on communication in VANET [8] is a work of realistic simulation of IEEE $802.11 \mathrm{p}$ channel, comparing propagation models in suburban scenarios of VANET using OPNET. Vehicle to vehicle communication is simulated in realistic wireless channel. The authors compared the Free space, Two ray ground and Dual slope piecewise linear path loss model with a Nakagami- $m$ channel model that they programmed to replace OPNET default free space propagation model. The Nakagami- $m$ parameter was varied as a function of velocity and separating distance. The results led to conclusion that complexity of propagation models can greatly affect the results obtained.

\section{SIMULATION SETUP}

In this study, for exploring and evaluating the protocol performance at variable speed in the presence of two different propagation models, simulations are carried out using Network Simulator-2 (Version 2.35).In order to investigate the QoS performance; two different scenarios are considered: one with vehicles moving at the speed of $10 \mathrm{~m} / \mathrm{s}$ and the other with vehicles at speed $20 \mathrm{~m} / \mathrm{s}$. The following table will summarize the simulation scenario considered.

Table 1. Simulation Parameters

\begin{tabular}{|l|l|}
\hline Network Simulator & NS-2 (Version 2.35) \\
\hline Wireless Terrain & $1200 x 1200$ \\
\hline Simulation Time & 10 min \\
\hline Routing Protocol & AODV,OLSR,ZRP \\
\hline Vehicle Density & 30 \\
\hline MAC & MAC 802_11 Ext \\
\hline PHY & WirelessPhyExt \\
\hline Backoff Algorithm at MAC & MBA \\
\hline Radio Propagation Model & TwoRayGround, Nakagami \\
\hline Data Traffic Source & UDP,CBR \\
\hline Packet Size & 1024 Bytes \\
\hline Modulation Scheme & BPSK \\
\hline Speed of Vehicle & $10 \mathrm{~m} / \mathrm{s}, 20 \mathrm{~m} / \mathrm{s}$ \\
\hline
\end{tabular}

Case 1: Vehicles moving at the speed of $10 \mathrm{~m} / \mathrm{s}$

Case 2: Vehicles moving at $20 \mathrm{~m} / \mathrm{s}$

5. RESULTS AND DISCUSSION

5.1. Case 1. With speed of $10 \mathrm{~m} / \mathrm{s}$

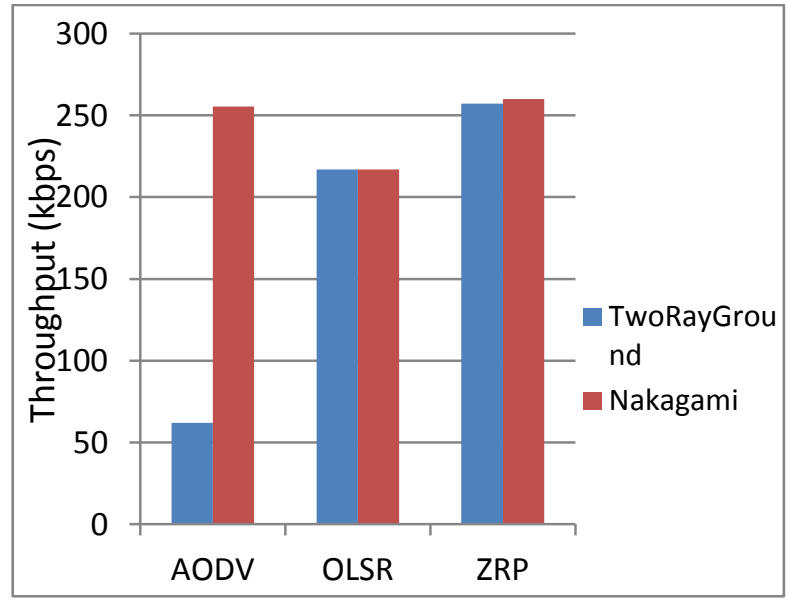

Fig. 1 Throughput of the protocols for the Propagation Models 
Figure 1 shows the Throughput of AODV, OLSR and ZRP. In case of AODV, there is variation in Throughput using both propagation models but it is almost constant for both OLSR and ZRP. Using Two Ray Ground, AODV has the lowest Throughput, OLSR has the average Throughput and ZRP has the highest Throughput. Using Nakagami, AODV has the highest Throughput followed by ZRP and OLSR.

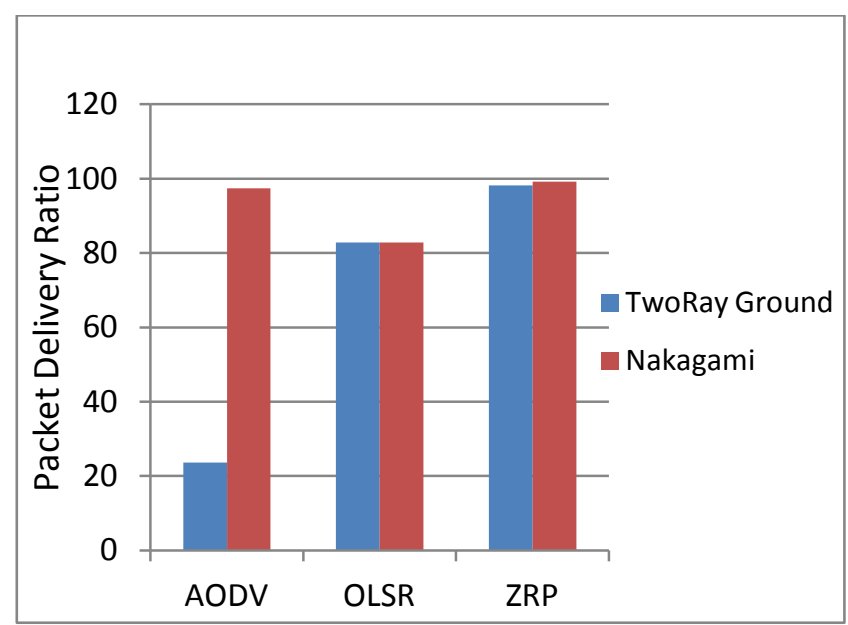

Fig. 2 Packet Delivery Ratio of the protocols for the Propagation Models

Figure above shows the PDR of AODV, OLSR and ZRP. Packet delivery ratio follows the same pattern as that for throughput. In case of AODV, there is variation in PDR using the two propagation models but it is almost constant for both OLSR and ZRP. Using Two Ray Ground, AODV has the lowest PDR, OLSR and ZRP both have the average PDR. Using Nakagami, all protocols have all most same PDR with little bit variations.

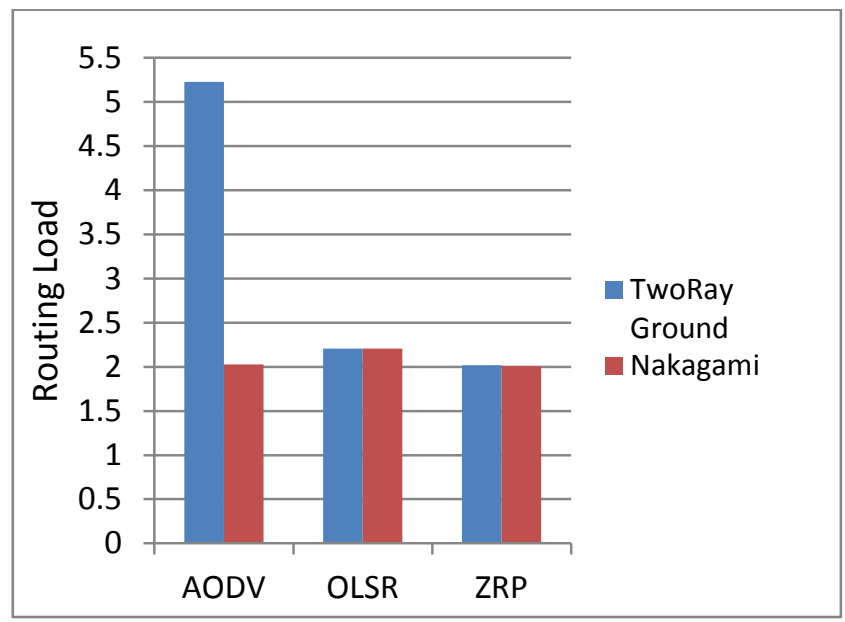

Fig. 3 Routing Load of the protocols for Two Ray Ground and Nakagami

Figure above describes the Routing Load of AODV, OLSR and ZRP. Using Two Ray Ground, AODV has the highest routing load as compared to OLSR and ZRP which have the average routing load. Using Nakagami, routing load is almost same with small variations.

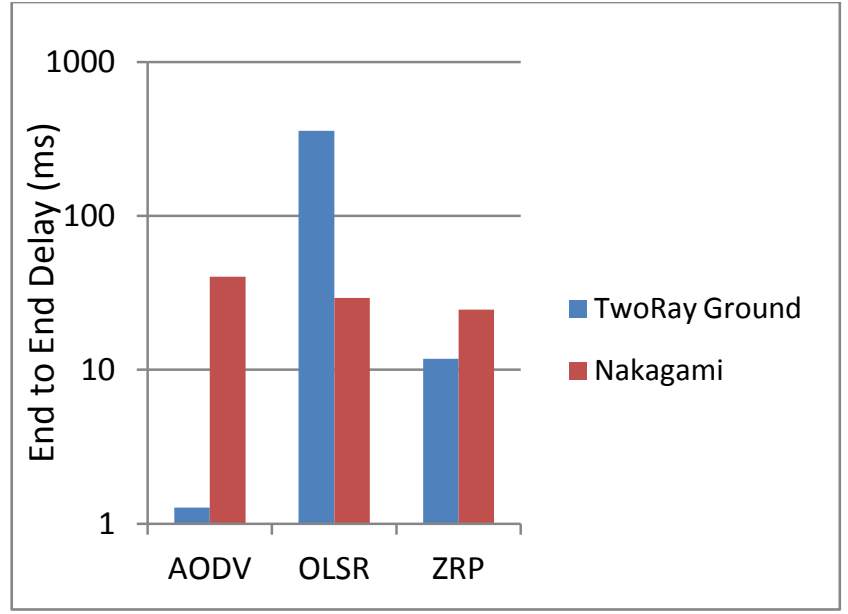

Fig. 4 End to End delay of the protocols with Two Ray Ground and Nakagami

Figure above illustrates the variations in the End-to-End Delay using different propagation Models at the node speed $10 \mathrm{~m} / \mathrm{s}$. As per the results we can observe that AODV has minimum value of the delay using Two Ray Ground where as ZRP has the average value of delay and OLSR has the highest value of Delay where as using Nakagami model AODV has the highest delay followed by the OLSR and ZRP.

\subsection{Case 2. With speed of $20 \mathrm{~m} / \mathrm{s}$}

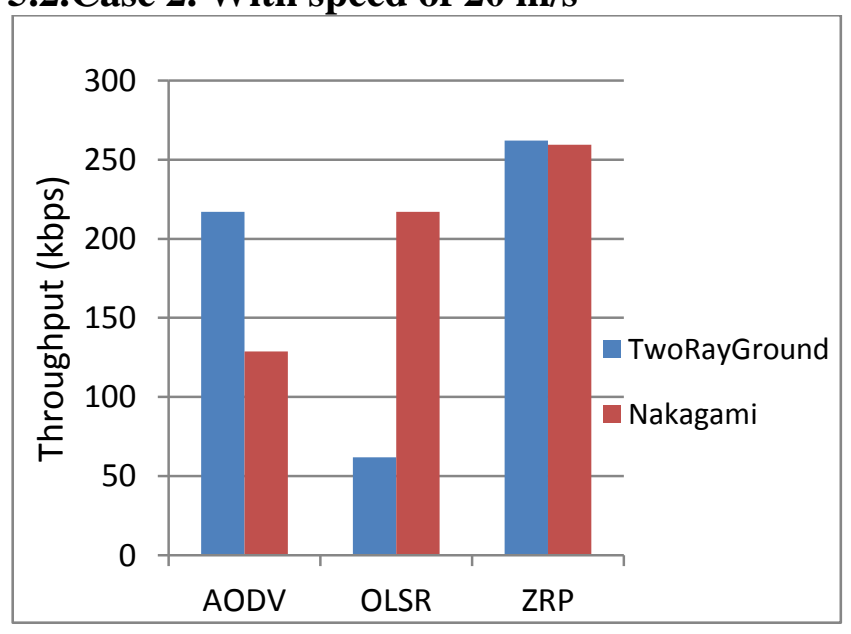

Fig. 5 Throughput of the protocols with the Propagation Models

Figure above shows the Throughput of AODV, OLSR and ZRP using different propagation models. Using Two Ray ground, ZRP has the highest Throughput followed by AODV and OLSR has the lowest Throughput. Using Nakagami, ZRP has the highest Throughput followed by OLSR and AODV has the lowest Throughput at node speed $20 \mathrm{~m} / \mathrm{s}$. 


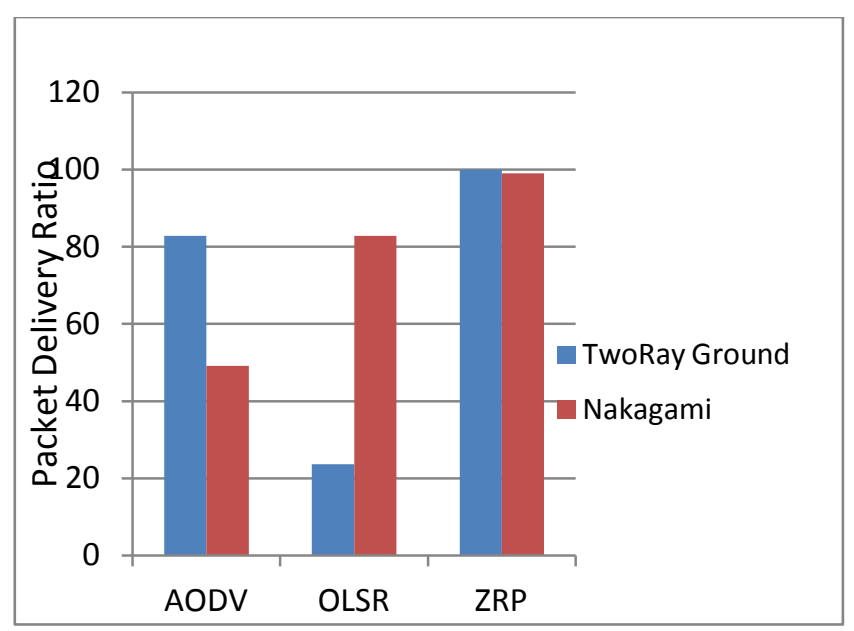

Fig. 6 Packet Delivery Ratio of the protocols with the Propagation Models

Figure 6 shows the PDR of AODV, OLSR and ZRP using different propagation models. Using Two Ray Ground model, ZRP has the highest PDR which is greater than AODV and OLSR which has the lowest PDR as compared to others. Using Nakagami model, ZRP has the highest PDR, PDR of OLSR is higher the AODV but less then ZRP and AODV has the minimum PDR as compared to others.

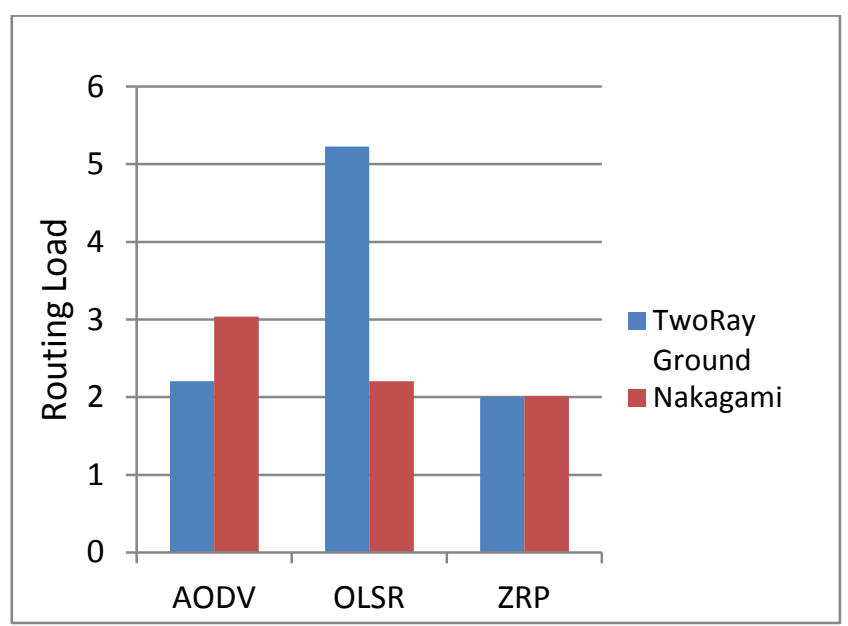

Fig. 7 Routing Load of the protocols using Two Ray Ground and Nakagami Propagation Models

Figure above displays the results obtained for the Routing Load of AODV, OLSR and ZRP using different propagation models. In case of Two Ray Ground, OLSR has the highest routing load followed by AODV and ZRP which has the lowest load. Using Nakagami model, AODV has the highest routing load, OLSR has medium routing load and ZRP has the lowest one.

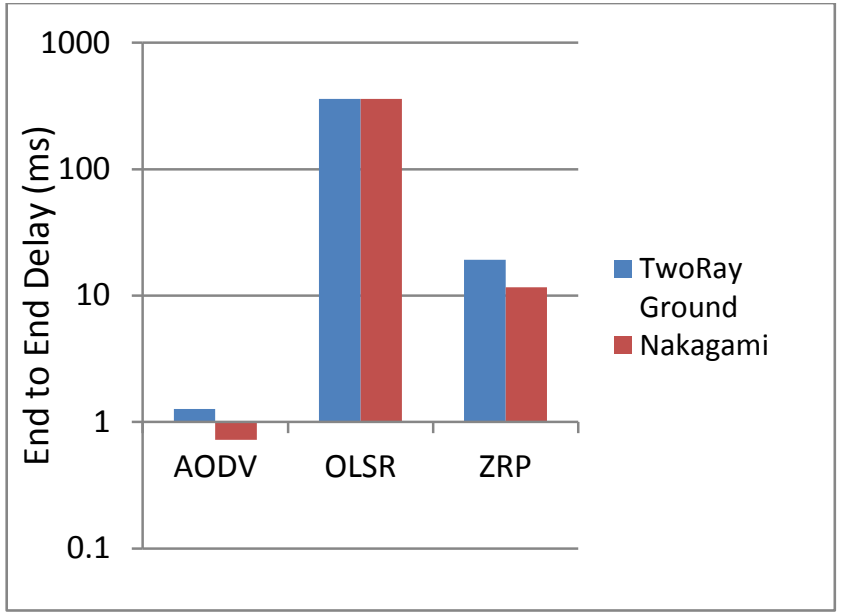

Fig. 8 End to end delay of the protocols using Two Ray Ground and Nakagami Propagation Models

Figure above show the variations in the End-to-End Delay using different propagation Models at the node speed $20 \mathrm{~m} / \mathrm{s}$. As per the results we can observe that AODV has minimum value of the delay where as ZRP has the average value of delay and OLSR has the highest value of Delay. Using Two Ray Ground, delay is more with AODV and ZRP but with OLSR both propagation models have all most same values for delay as compared to Nakagami propagation model.

\section{CONCLUSION}

The main goal of this paper is to determine the effect of speed and radio propagation model on the operation and performance of routing protocols that fall in reactive, proactive and hybrid category. The QoS parameters are examined for each of the protocol considered. At low speed of $10 \mathrm{~m} / \mathrm{s}$, In case of Two Ray Ground, Throughput and PDR of AODV is the lowest, followed by OLSR and ZRP has the highest throughput and PDR. Routing load is very high for AODV. OLSR and ZRP have nearly similar values of load. End to end delay is the highest for OLSR followed by ZRP and lowest for AODV. In case of Nakagami, the throughput and PDR of AODV improves. OLSR and ZRP have similar behaviour as that of Two Ray Ground. With the increase in speed i.e. at $20 \mathrm{~m} / \mathrm{s}$ there are variation in the values of all the protocols using both Two Ray Ground and Nakagami. With the increase in speed the throughput and PDR of the protocols decrease in case of Nakagami Propagation and there are variations using Two Ray ground. It can be concluded that mobility and radio propagation models are critical in designing and evaluating VANETs and considerably effect the performance of protocols so should be carefully chosen to reflect reality.

\section{ACKNOWLEDGEMENTS}

I express my gratitude to honourable Dr. Jyoteesh Malhotra for his guidance and direction which kept me focussed throughout this venture and helped me complete this work. I am also very grateful to the Almighty for giving me strength and keeping me on to the right track. 


\section{REFERENCES}

[1] Ramesh C. Poonia and Vikram Singh,"Performance Evaluation Of Radio Propagation Model for Vehicular Ad Hoc Networks using VANETMOBISIM and NS-2", International Journal of Distributed and Parallel Systems (IJDPS) Vol.3, No.4, July 2012, DOI : 10.5121/ijdps.2012.3415 145 .

[2] Pranav Kumar Singh, "Influences of TwoRayGround and Nakagami Propagation model for the Performance of Adhoc Routing Protocol in VANET", International Journal of Computer Applications (0975 - 8887) Volume 45- No.22, May 2012.

[3] Pranav Kumar Singh, Kapang Lego,"Comparative Study of Radio Propagation and Mobility Models in Vehicular Adhoc Network", International Journal of Computer Applications (0975 - 8887) Volume 16- No.8, February 2011

[4] Javier Gozalvez, Miguel Sepulcre, Ramon Bauza," Impact of the radio channel modelling on the performance of VANET communication protocols", Springer Science+Business Media, LLC 2010, Telecommun Syst DOI 10.1007/s11235-010-9396.

[5] Henrik Schumacher and Hugues Tchouankem," Highway Propagation Modeling in VANETs and Its Impact on Performance Evaluation”, 2013 10th Annual Conference on Wireless On-demand Network Systems and Services (WONS)
[6] Francisco J. Martinez, Chai-Keong Toh, Juan-Carlos Cano, Carlos T. Calafate, Pietro Manzoni," Realistic Radio Propagation Models (RPMs) for VANET Simulations", WCNC 2009 proceedings, IEEE.

[7] Bilal Munir Mughal, Asif Ali Wagan , Halabi Hasbullah," Analyzing Safety Beacon Loss Rate in VANETs with Two-Ray Ground and Nakagami Propagation Models", 978-1-4577-1884-7/2011 IEEE.

[8] Tarikul Islam, Yongchang Hu, Dr. Ertan Onur, Dr. Bert Boltjes, Dr. J.F.C.M de Jongh," Realistic Simulation of IEEE 802.11p Channel in Mobile Vehicle to Vehicle Communication", 13th Conference on Microwave Techniques COMITE 2013, April 17-18, Pardubice, Czech Republic.

[9] Aarja Kaur, Jyoteesh Malhotra," On the Selection of Efficient Backoff with QoS Aware Routing in VANET", International Journal of Applied Mathematics, Electronics and Computers [Communicated].

[10] Saif Al-Sultan , Moath M. Al-Doori, Ali H. Al-Bayatti, Hussien Zedan," A comprehensive survey on vehicular Ad Hoc network", Journal of Network and Computer Applications www.elsevier.com/locate/jnca, 21 February 2013. 